\title{
Tetraspanins CD9 and CD151, epidermal growth factor receptor and cyclooxygenase-2 expression predict malignant progression in oral epithelial dysplasia
}

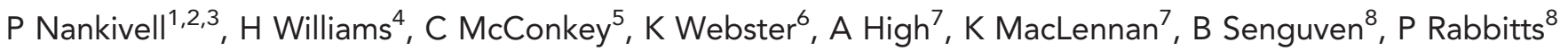
and Hehanna*,1,3

${ }^{1}$ Institute of Head and Neck Studies and Education (InHANSE), School of Cancer Sciences, University of Birmingham, Birmingham B15 2TT, UK; ${ }^{2}$ Department of Otolaryngology, University Hospital Coventry and Warwickshire, Coventry CV2 2DX, UK; ${ }^{3}$ Department of Otolaryngology, University Hospital Birmingham, Birmingham B15 2WB, UK; ${ }^{4}$ Department of Histopathology, University Hospital Birmingham, Birmingham B15 2WB, UK; ${ }^{5}$ Clinical Trials Unit, Warwick Medical School, University of Warwick, Coventry CV4 7AL, UK; ${ }^{6}$ Department of Maxillofacial Surgery, University Hospital Birmingham, Birmingham B15 2WB, UK; ${ }^{7}$ Department of Histopathology, Leeds Teaching Hospitals Trust, Leeds LS7 4SA, UK and ${ }^{8}$ Section of Pre-Cancer Genomics, Leeds Institute of Cancer Studies and Pathology, University of Leeds, Leeds LS9 7TF, UK
\end{abstract}

Background: Prognostic biomarkers aim to improve on the current inadequate method of histological assessment to identify patients with oral epithelial dysplasia at greatest risk of malignant transformation. We aimed to assess the prognostic ability of six protein biomarkers linked to the epidermal growth factor receptor (EGFR) pathway, including three tetraspanins, in a large multicentre oral dysplasia cohort.

Methods: One hundred and forty-eight cases with varying degrees of epithelial dysplasia underwent immunohistochemical assessment for CD9, CD151, CD82, EGFR, Her-2, and COX-2. Scoring was performed independently by two observers. Univariate analyses using both logistic and Cox regression models and a multivariate regression were performed.

Results: Malignant progression was significantly greater in those cases with decreased expression of CD9 $(P=0.02)$, and increased expression of CD151 ( $P=0.02)$, EGFR $(P=0.04)$, and COX-2 $(P=0.003)$. Histological grade $(P=0.0002)$ and morphology $(P=0.03)$ were also prognostic, whereas smoking and alcohol were not. The optimal combination by backward-variable selection was of histological grade (hazard ratio (HR) 1.64; 95\% Cl 1.12, 2.40), COX-2 overexpression (HR 1.12; 1.02, 1.24) and CD9 underexpression (HR 0.88; 0.80, 0.97). CD82 and Her-2 demonstrated no prognostic ability.

Conclusion: This is the first study of the expression and prognostic potential of the tetraspanins in oral dysplasia. A combination of certain biomarkers with clinical factors appeared to improve the accuracy of determining the risk of malignancy in individuals with oral dysplasia. These findings may also offer potential new therapeutic approaches for this condition.

Cancers of the oral cavity arise through a combination of progressive genomic alteration and exposure to environmental carcinogens (Califano et al, 1996). Many OSCCs arise in areas of genomic and histological abnormality, termed as oral epithelial dysplasia (OED). The degree of cytological and architectural abnormality seen on histological examination is used to assign a

*Correspondence: Professor H Mehanna; E-mail: h.mehanna@bham.ac.uk

Received 17 May 2013; revised 13 August 2013; accepted 11 September 2013; published online 7 November 2013

(c) 2013 Cancer Research UK. All rights reserved 0007-0920/13 
grade of severity to OED (Barnes et al, 2005; Warnakulasuriya et al, 2008). Quantifying the risk of transformation of an individual OED lesion to cancer is complex, due to both a lack of knowledge of the natural history of OED and the wide variability in reported transformation rates in the published literature (5-36\%) (Silverman et al, 1984; Hsue et al, 2007). A recent meta-analysis estimated the malignant transformation of OED to be $12 \%$; $95 \%$ CI 8-18\% (Mehanna et al, 2009). Furthermore, while dysplasia grade assessed by histological examination is currently the best predictor of future malignant behaviour, it has significant limitations. Despite more severe grades of dysplasia being associated with higher transformation rates, cases with mild dysplasia may still progress to cancer, while a significant proportion with severe dysplasia do not transform, irrespective of environmental factors (Barnes et al, 2005; Warnakulasuriya et al, 2008; Mehanna et al, 2009; Ferlay et al, 2010; Liu et al, 2011). In addition, histological grading of OED is known to be largely subjective, resulting in a significant inter- and intra-rater variability (Abbey et al, 1995; Karabulut et al, 1995; Tabor et al, 2003; Fischer et al, 2004). This results in histological grading having only a moderate prognostic ability at best. However to date, it remains the gold standard on which treatment decisions are based (Nankivell and Mehanna, 2011).

The differential expression of biomarkers in cancer, potentially malignant lesions and normal mucosa offers the possibility of better identification of those lesions with the highest risk of malignant progression. To date, many biomarkers have been studied in OED, with only a minority showing any prognostic potential. These include loss of heterozygosity at the $3 p \pm 9 p$ loci (LOH), increased levels of survivin and matrix metalloproteinase- 9 mRNA, allelic index and DNA content (Hogmo et al, 1998; Rosin et al, 2000; Muzio et al, 2003; Zhou et al, 2005; Smith et al, 2009). Yet due to low sample size and methodological limitations, only $\mathrm{LOH}$ has been validated and none have as yet been incorporated into routine clinical use (Zhang et al, 2012). The search for effective prognostic biomarkers continues.

The epidermal growth factor receptor (EGFR) family has been extensively studied in relation to cancer biology. Strong evidence exists for their role in carcinogenesis in many solid tumours, including those arising in the breast, ovary, colon and lung (Normanno et al, 2008). Overexpression of EGFR occurs in around $80-90 \%$ of head and neck cancers and in some studies has been shown to be correlated with worse survival outcomes (Grandis and Tweardy, 1993; Etienne et al, 1999; Ang et al, 2002). Another of the EGF family, Her2 is similarly upregulated in oral dysplasia and cancer (Silverman et al, 1984; Hsue et al, 2007; Rautava et al, 2008). This pathway is also of interest, as molecular therapies targeted against EGFR already exist. These include small molecule tyrosine kinase inhibitors (e.g., gefitinib) and monoclonal antibodies (e.g., cetuximab), which may potentially be of benefit for the treatment of OED. Modulation of EGFR signalling is known to occur by several mechanisms. Two of these mechanisms involve cyclooxygenase enzymes and tetraspanin proteins. Tetraspanins are a family of 33 proteins that when joined by gangliosides and cholesterol form aggregations termed tetraspanin-enriched microdomains (Zöller, 2009). Through these domains, tetraspanins are involved in the lateral coordination of other transmembrane molecules including growth factor receptors (Abbey et al, 1995; Karabulut et al, 1995; Tabor et al, 2003; Fischer et al, 2004; Murayama et al, 2008), integrins (Berditchevski, 2001), and G-protein coupled receptors (Little et al, 2004; Normanno et al, 2008; Zöller, 2009). The modulatory abilities of these tetraspanins result in changes to tumorigenic processes, such as cell adhesion, motility, invasion, and angiogenesis (Hemler, 2005; Zöller, 2009). Many of the functions of the tetraspanin proteins remain unclear, with only a few of the 33 proteins in this family having been intensively studied. This has not been aided by investigations focussing on the functionality of individual tetraspanins, despite evidence for their ability to form and probably function as complexes (Lazo, 2007). Three of the tetraspanins (CD9, CD151, and CD82) have been shown to interact with EGFR, either directly or in conjunction with integrin adhesion receptors (Wang et al, 2007; Park et al, 2009; Deng et al, 2012; Romanska et al, 2012; Odintsova et al, 2013). Furthermore, there is evidence that reduced expression of CD9 (Mhawech et al, 2004; Buim et al, 2010) and CD82 (Imai et al, 2002; Farhadieh et al, 2004), and increased expression of CD151 (Hirano et al, 2009) confers a poor prognosis in head and neck cancer. It is possible, therefore, that these tetraspanins may also have a role earlier in the carcinogenic process. However, their prognostic effect, either alone or in conjunction with EGFR, has not been examined in OED.

Our aim was to examine the prognostic potential of EGFR and biomarkers known to modulate this pathway, in conjunction with clinical factors, in one of the largest cohorts of OED reported in the literature.

\section{MATERIALS AND METHODS}

This study has been reported using the REporting recommendations for tumour MARKer prognostic studies (REMARK) guidelines (McShane et al, 2005). Ethical approval was granted from the Coventry research ethics committee (06/Q2802/79) and the Human Biomaterials Resource Centre at the University of Birmingham (10-008).

Patient selection. This was a retrospective cohort study. Consecutive cases were selected after systematic searching of the pathology archives from five institutions: University Hospital Coventry and Warwickshire, University Hospital Birmingham, Birmingham Dental Hospital, George Eliot Hospital Nuneaton, and St James University Hospital, Leeds. Searching was performed using the Systematized Nomenclature of Medicine Clinical Terms (SNOMED) and free field text, to include any biopsies taken between 1996 and 2008. Inclusion in the cohort required patients to be over 18 years of age at time of biopsy (no upper age limit was set), have a confirmed diagnosis of OED using the WHO classification system, and have a minimum follow-up for nontransformed cases of 12 months, or transformation to cancer after 3 months of diagnosis of OED. Where several biopsies were available from a single patient, the first diagnostic biopsy was used. Where the first diagnostic biopsy was not available, the next oldest biopsy was used. Cases were excluded if positive for Candida on diastase-resistant periodic acid schiff (dPAS) staining, along with diagnoses of lichenoid inflammation with atypia (histological changes are likely a result of inflammation and therefore represents a different process to true neoplastic change) and proliferative verrucous leukoplakia. Any patient with OED that had a previous diagnosis of head and neck cancer (identified either through the pathology database or a search of the clinical records) was excluded, as this population of patients is known to already be at increased risk of developing a second malignancy, and previous treatment may have affected the behaviour of the lesion under investigation. Clinical information on the exposure to known or suspected risk factors such as age, gender, anatomical site, lesion morphology, and smoking/alcohol history were collected.

Cohort characteristics. One hundred and forty-eight patients with OED were included in this cohort. They were almost equally divided between male (76) and female (72). The mean age was 61 years (SD 13.6) with a range from 19 to 90. Other demographic data including OED dysplasia grade are summarised in Table 1. Thirty-nine out of one hundred and forty-eight cases progressed from dysplasia to cancer (26\%) with a median time to transformation for these cases of 26 months. 
Table 1. Clinical characteristics of cohort (number and percent) with prognostic ability by univariate logistic regression ( $P$-value)

\begin{tabular}{|c|c|c|}
\hline Histological grade & & 0.0002 \\
\hline Mild & $69(47)$ & \\
\hline Moderate & $50(34)$ & \\
\hline Severe & $27(18)$ & \\
\hline $\mathrm{CIS}$ & $2(1)$ & \\
\hline Gender & & 0.14 \\
\hline Male & $76(51)$ & \\
\hline Female & $72(49)$ & \\
\hline Site of lesion & & 0.73 \\
\hline Tongue & $69(47)$ & \\
\hline Floor of mouth & $20(13)$ & \\
\hline Palate & $18(12)$ & \\
\hline Buccal & $38(26)$ & \\
\hline Retromolar & $3(2)$ & \\
\hline Morphology of lesion & & 0.03 \\
\hline White patch & $94(63)$ & \\
\hline Red patch & $15(10)$ & \\
\hline Speckled patch & $13(9)$ & \\
\hline Ulcer & $22(15)$ & \\
\hline Lump & $4(3)$ & \\
\hline Alcohol consumption & & 0.61 \\
\hline$>21 \mathrm{U} /$ week & $23(15)$ & \\
\hline$<21 \mathrm{U} /$ week & $58(40)$ & \\
\hline None & $44(30)$ & \\
\hline Unknown & $23(15)$ & \\
\hline Smoking status & & 0.29 \\
\hline Current & $69(47)$ & \\
\hline Ex & $9(6)$ & \\
\hline Non & $47(32)$ & \\
\hline Unknown & $23(15)$ & \\
\hline
\end{tabular}

It is possible that the findings of differential biomarker expression between cases transforming and not transforming in this study may differ depending on the Human Papilloma Virus (HPV) status. p16 ${ }^{\mathrm{INK} 4 \mathrm{a}}$ expression as a surrogate for HPV status has been examined as a part of another study in this cohort. Only $7 \%$ of cases were positive for $\mathrm{p} 16^{\mathrm{INK} 4 a}$ and therefore the results of this current study should be viewed in light of it being a largely HPV-negative cohort (submitted but unpublished work by the same author group).

Immunohistochemistry. All samples were taken from formalinfixed, paraffin-embedded tissue. Four micrometre sections were taken either from donor blocks or from a tissue microarray containing some of the cases. We have previously demonstrated near perfect agreement in immunohistochemical scoring between tissue microarrays and slides using these biomarkers in OED (Nankivell et al, 2012). After deparaffinisation in xylene, sections were rehydrated in distilled water. Unmasking of the epitopes was performed using a PickCell antigen retrieval unit (PickCell Laboratories, Leiden, The Netherlands), exposing the samples to both heat and pressure while in Tris-EDTA buffer concentrate at $\mathrm{pH}$ 7.8, or Citrate buffer $\mathrm{pH} 6$ (determined by prior optimisation and validation). The Novocastra Polymer Detection System (Leica Biosystems Newcastle Ltd, Newcastle Upon Tyne, UK) was used for this study. Endogeneous peroxidase and protein was blocked with 3\% hydrogen peroxide and $0.4 \%$ Casein in phosphate-buffered saline, respectively. Slides were then incubated at $4{ }^{\circ} \mathrm{C}$ with monoclonal antibodies at optimal concentrations
(Supplementary Table 1). After $30 \mathrm{~min}$ incubations with post primary block and polymer, 3,3'-Diaminobenzidine (DAB) working solution was applied for $5 \mathrm{~min}$. Application of Mayer's haematoxylin for $1 \mathrm{~min}$ provided counterstaining. All positive controls stained correctly and no negative controls demonstrated any staining during the procedure.

Immunohistochemical scoring. Two individual raters, with different levels of experience in immunohistochemistry assessment, independently scored each case. These assessors were blinded to the clinical details of the case. It has been suggested that when scoring immunoreactivity in small specimens (such as OED specimens examined here) only the area with maximal staining should be interpreted (Goldstein and Bosler, 2007). This approach was applied here. The sections were presented in random order to the raters with cases of disagreement undergoing consensus scoring. Antibody expression was determined by assessing the intensity and proportion of cells stained. Staining intensity was scored from 0 to 3: $0=$ negative (No staining); $1=$ weak staining; $2=$ moderately strong staining; and $3=$ strong staining. Proportion was also scored on a 4 -point scale: 1 ( $<25 \%$ of cells stained); $2(25-50 \%$ of cells stained); $3(51-75 \%$ of cells stained); and 4 ( $>75 \%$ cells stained). An overall score for each case was generated by the sum of the intensity and proportion scores, resulting in a range of scores from 0 to 12 .

Statistical analysis. Scoring agreement between raters was calculated using a kappa statistic $(\kappa)$ and intraclass correlation coefficients (ICCC). The latter measure is felt to be superior when correlating immunohistochemistry scores between raters, as it is calculated using the whole range of data, thereby not being influenced on how the data are categorised, as is the case with kappas (Kirkegaard et al, 2006). However, many studies quote kappa scores and hence they were also included here to allow comparison. Kappa scores were interpreted using a scale proposed by Landis and Koch (1977), with scores of 0-0.2 representing slight, 0.2-0.4 fair, 0.4-06 moderate, 0.6-0.8 substantial, and 0.8-1.0 near perfect agreement. An ICCC of $<0.40$ was regarded as poor, $0.4-0.59$ as fair, $0.6-0.74$ as good, $>0.74$ as excellent, and 1 as perfect correlation (Carrasco and Jover, 2003). Consensus scores between the two raters were used for subsequent analyses. The capability of each biomarker or clinical factor to predict progression was initially calculated using univariate logistic regression. Consideration of the additional effect of time on the prognostic ability was assessed using a Cox regression analysis, with significance defined as $P<0.05$. Clinical factors were analysed as categorical variables as shown in Table 1. Missing data were handled using listwise deletion, where any cases with missing clinical data were excluded from the analysis of that particular variable. Multivariate analysis with backward-variable selection was performed to examine which factors remained independent indicators of transformation. This method was chosen in preference to a forward selected model, which may sometimes miss out some interesting variables.

To further explore the scoring thresholds that predict progression, a logistic regression was performed on the continuous immunohistochemistry scores. Where a linear effect was not seen, scores were then converted into categorical variables to examine whether prognostic ability differed between these categories. Categorisation was as follows: score of $0=0$ (truly negative), scores of $1-4=1$ (weakly positive), 5-8 =2 (moderately positive), and $>9=3$ (strongly positive). Finally, Pearson's chi-squared analysis was used to identify the optimal binary scoring threshold to group cases into the most and least likely to transform. Oral cancer-free survival was calculated for these different groups using Kaplan-Meier survival curves. Differences between the resulting curves were calculated using a Log-rank (Mantel-Cox) test. 
Calculations were performed using SPSS version 19.0 for Mac; SPSS Inc., Chicago, IL, USA and SAS version 9.2.

\section{RESULTS}

Inter-rater scoring reliability. There was strong agreement between the two raters for all biomarkers used in this study. Kappa scores ranged from 0.66 to 1.0 demonstrating substantial agreement. This finding was confirmed with ICCC ranging from 0.82 to 1.0 (Supplementary Table 2). The most variability was seen on scoring the intensity of COX2 staining ( $\kappa 0.66$; ICCC 0.85$)$ and proportion of EGFR cells stained ( $\kappa 0.74$; ICCC 0.82$)$. However, this still represents substantial agreement.

Prognostic ability of clinical factors. Using a univariate logistic regression, higher grades of dysplasia were seen to significantly predict malignant transformation in this cohort $(P=0.0002)$. This remained significant when time to transformation was analysed using a Cox regression model $(P=0.001)$. The morphology of the individual lesions was also associated with progression $(P=0.03)$. In ascending order, the proportion of progressors for each morphological type was leukoplakia (17 out of 94,18\%), ulcerated lesions ( 7 out of $22,32 \%$ ), speckled lesions ( 5 out of $13,38 \%$ ), mass lesions ( 2 out of $4,50 \%$ ), and erythroplakia (8 out of 15,53\%). However, morphology did not remain independently significant once added to grade in a multivariate analysis. Anatomical site, smoking, and alcohol consumption were not prognostic $(P=0.73$, 0.29 , and 0.61 , respectively). Gender did not independently predict progression, yet showed a trend towards significance when added with histological grade into the multivariate model, with females more at risk than males $(P=0.05)$.

Prognostic ability of biomarkers. Immunohistochemical expression of each of the biomarkers is summarised in Supplementary Table 3. The pattern of staining was predictable,
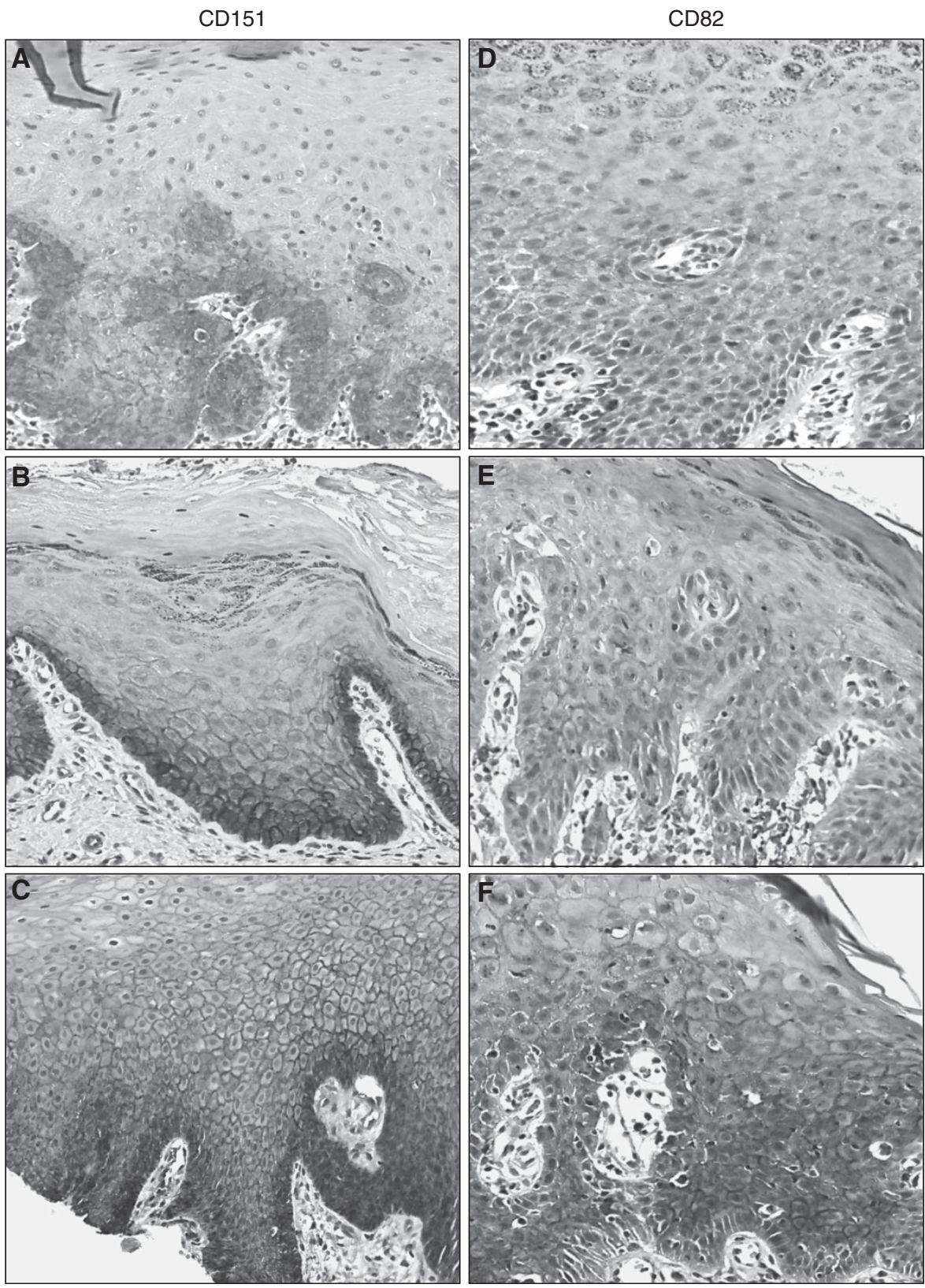

Figure 1. Representative tetraspanin immunohistochemistry. Tiles (A-C) (CD151) and (D-F) (CD82) demonstrate increasing expression from scores of 2 (top row) to 6 (middle row) to 12 (bottom row). Both tetraspanin biomarkers exhibit membranous staining. 

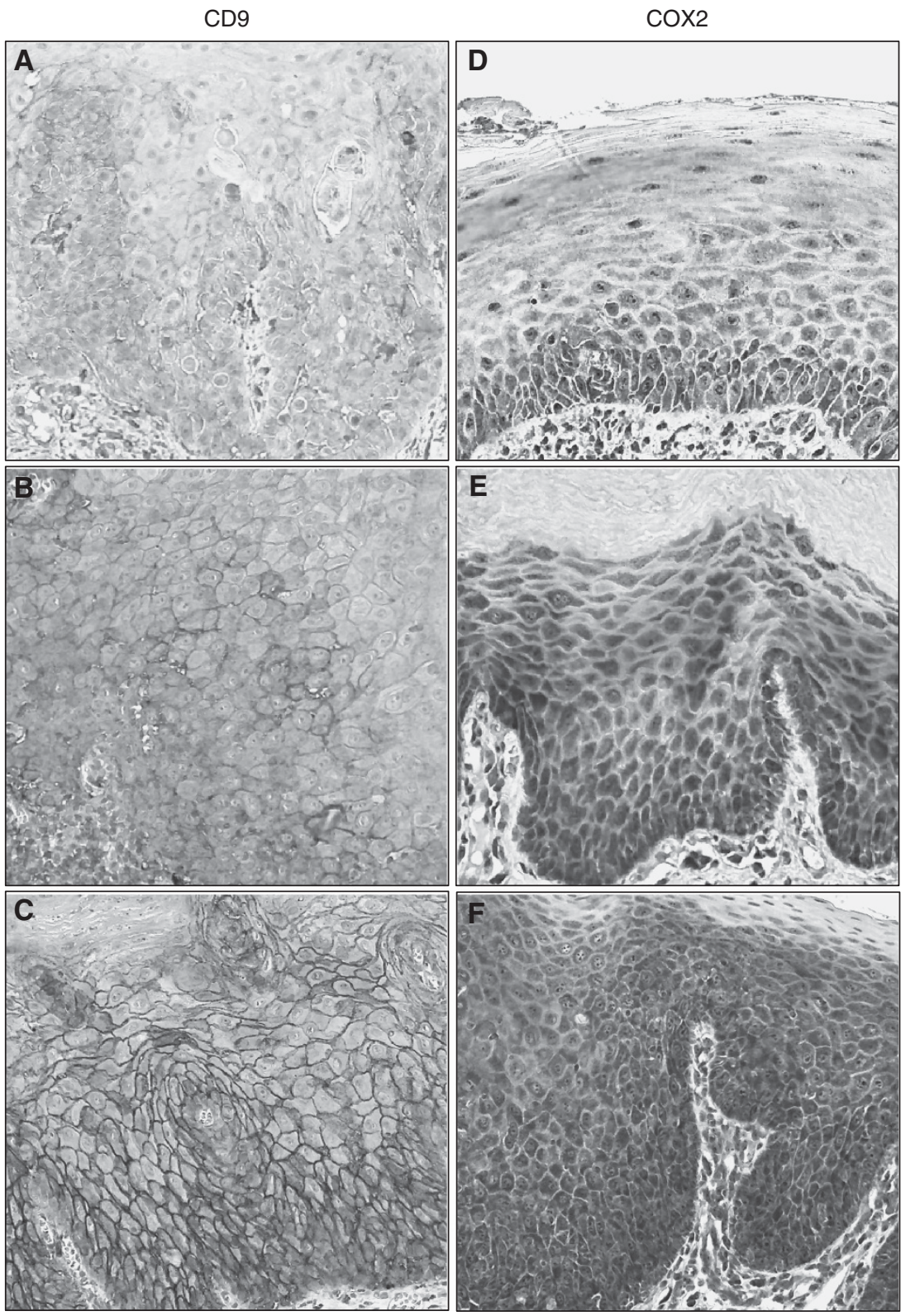

Figure 2. Representative immunohistochemistry. Tiles (A-C) (CD9) and (D-F) (COX2) display increasing expression from scores of 2 (top row), to 6 (middle row) to 12 (bottom row). CD9 demonstrates membranous staining, whereas COX2 shows predominantly cytoplasmic staining.

with CD9, CD151, CD82, and EGFR localising to the cell membrane, and COX2 to the cytoplasm (Figures 1-3). There was also a small amount of nuclear staining evident with increased COX2 expression. Only 8 out of the 148 cases demonstrated any Her2 staining, with all of these being membranous in location. The remaining Her2 cases had vague cytoplasmic and occasional nuclear staining, not considered to be representative of true Her2 protein expression (Figure 3d). Cytoplasmic/nuclear Her2 staining, along with nuclear COX2 staining, was not evaluated by the assessors when scoring. Nearly $80 \%$ of cases had very weak or no CD82 staining (scores $\leqslant 3$ ). Both raters agreed that scoring was not possible in 4 out of 888 slides $(0.5 \%)$ because of inadequate tissue.

Univariate logistic regression demonstrated a significantly increased risk of progression to cancer in cases with underexpression of CD9 $(P=0.02)$ or overexpression of CD151 $(P=0.02)$, EGFR $(P=0.04), \quad$ or $\mathrm{COX} 2 \quad(P=0.003)$. When also considering time to transformation, CD9 $(P=0.02)$, EGFR
$(P=0.04)$, and COX2 $(P=0.008)$ were still able to significantly predict progression (Table 2). On multivariate analysis, CD9 $(P=0.009)$ and $\mathrm{COX} 2(P=0.008)$ remained significant independent predictors of transformation to oral cancer. Epidermal growth factor receptor was not independently significantly associated with transformation on multivariate analysis.

Logistic regression was performed in an attempt to more accurately define relevant scoring thresholds for the biomarkers with prognostic potential. COX2 was the only marker to demonstrate a clear linear effect, with increasing scores associated with increasing risk of malignant progression $(P=0.002$, Figure 4$)$. No linear effect was seen with the other markers, even after the continuous scores $(0-12)$ were converted into categorical variables. Pearson's chi-squared analysis identified the optimal scoring thresholds to divide cases into those most and least likely to undergo malignant transformation. For CD9 and CD151, the threshold was between those cases scoring 

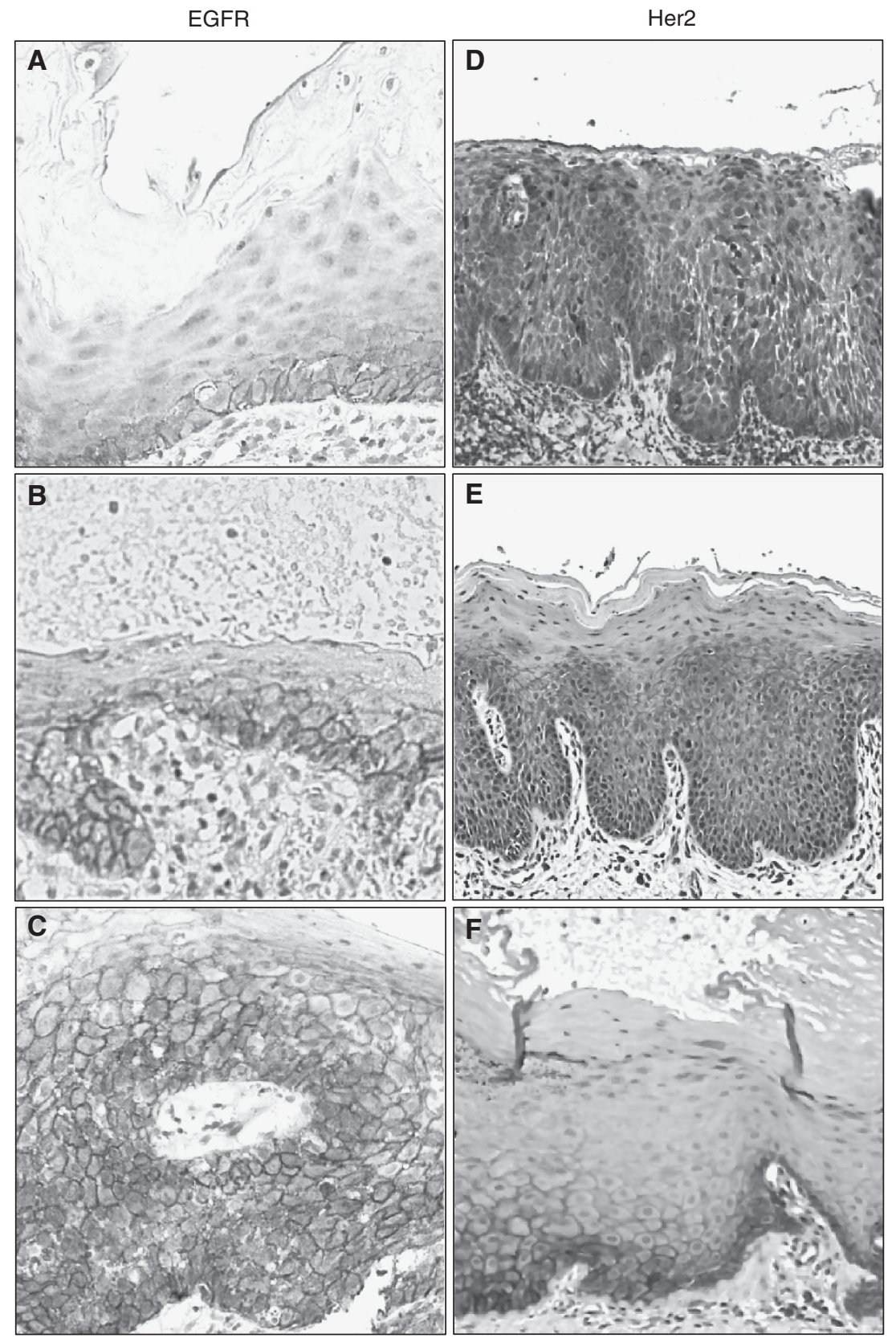

Figure 3. Representative immunohistochemistry. Tiles (A-C) (EGFR) and (D-F) (Her2) display increasing expression from scores of 2 (top row), to 6 (middle row) to 12 (bottom row). The strongest her 2 staining was scored as 3 out of 12 (F). Some cases demonstrated both cytoplasmic and membranous staining $(\mathbf{E})$ and were considered as positive. Where only cytoplasmic staining occurred (D) this was considered as negative and given a score of 0 .

Table 2. Prognostic ability of individual biomarkers on univariate analysis using logistic and Cox regression ( $P$-values)

\begin{tabular}{|l|c|c|}
\hline Biomarker & $\begin{array}{c}\text { Univariate } \\
\text { analysis } \\
\text { (Logistic) }\end{array}$ & $\begin{array}{c}\text { Univariate } \\
\text { analysis } \\
\text { (Cox) }\end{array}$ \\
\hline COX2 & 0.003 & 0.008 \\
\hline CD9 & 0.02 & 0.02 \\
\hline CD151 & 0.02 & 0.33 \\
\hline EGFR & 0.04 & 0.04 \\
\hline CD82 & 0.62 & 0.69 \\
\hline Her2 & 0.73 & 0.50 \\
\hline
\end{tabular}

0 or 1 vs the rest $(2-12) \quad(P<0.0001$ and 0.0002 , respectively), and $0-2 \quad v s$ the rest $(3-12)$ for $\operatorname{EGFR}(P=0.006)$ (Figure 4).

Because CD9 has been postulated to have an action via direct effects on EGFR expression, any association between these markers was explored. The correlation was low (Pearson's correlation coefficient $0.04, P=0.61$ ), showing no evidence of an association.

Prognostic ability of clinical factors and biomarkers. In combining both the clinical factors and biomarkers, the overall best combination by backward-variable selection was high dysplasia grade (hazard ratio (HR) 1.64; 95\% CI 1.12, 
A

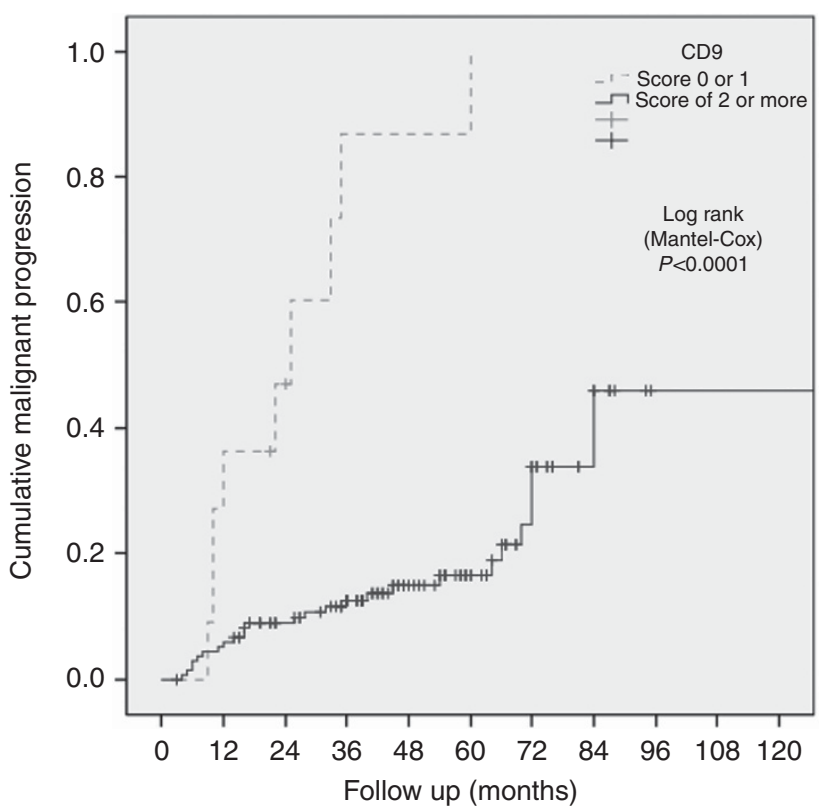

C

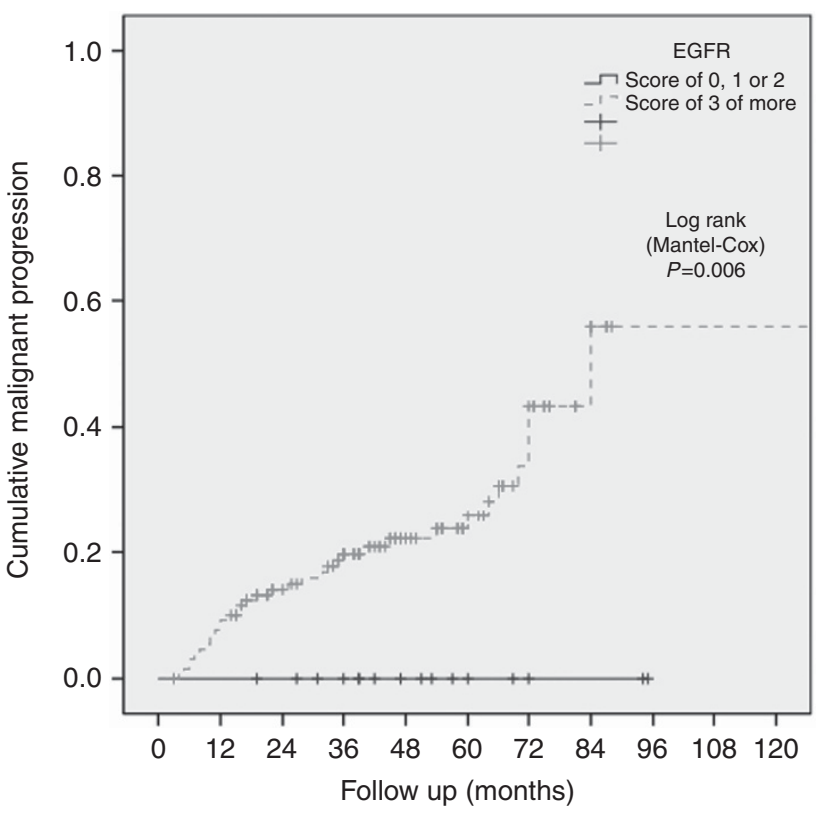

B

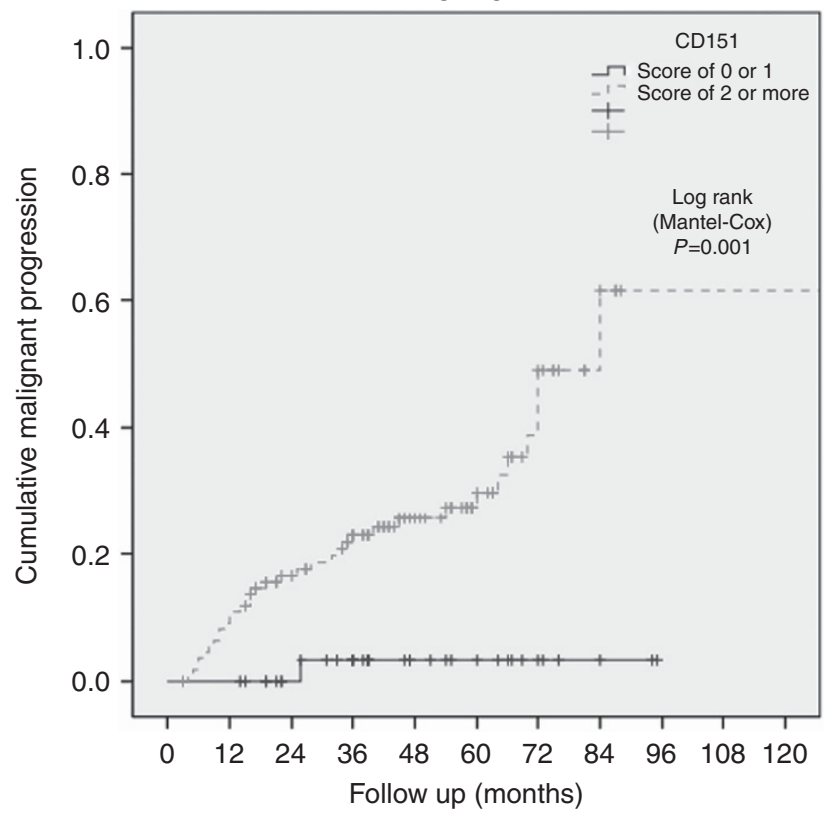

D

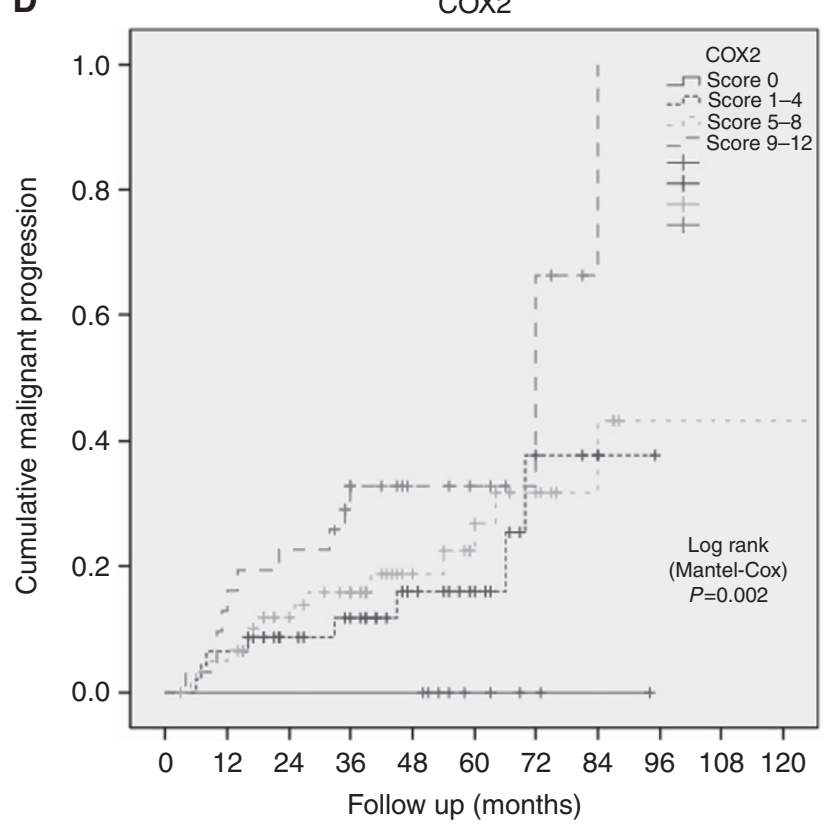

Figure 4. (A-D) Oral cancer-free survival utilising different ordered scoring thresholds for CD9, CD151, EGFR, and COX2.

Table 3. Multivariate analysis demonstrating hazard ratios for the best combination of clinical factors and biomarkers in predicting malignant progression by backward-variable selection

\begin{tabular}{|l|c|c|c|}
\hline Variable & $\begin{array}{c}\text { Hazard ratio } \\
(\mathbf{9 5 \%} \mathrm{Cl})\end{array}$ & $\boldsymbol{P}$-value & $\begin{array}{c}\text { Higher risk } \\
\text { group }\end{array}$ \\
\hline Grade & $1.64(1.12,2.40)$ & 0.01 & High grade \\
\hline COX2 & $1.12(1.02,1.24)$ & 0.02 & High score \\
\hline CD9 & $0.88(0.80,0.97)$ & 0.01 & Low score \\
\hline Abbreviation: $\mathrm{Cl}=$ confidence interval. \\
\hline
\end{tabular}

2.40, $P=0.01$ ), COX2 overexpression (HR 1.12; 95\% CI 1.02, 1.24, $P=0.02$ ), and underexpression of CD9 (HR $0.88,95 \%$ CI $0.80,0.97, P=0.01$ ) (Table 3 ).

\section{DISCUSSION}

This is the first study to examine the expression of members of the tetraspanin family in OED and the first to demonstrate a prognostic ability of CD9, CD151, COX2, and EGFR in a retrospective longitudinal OED cohort. Decreased expression of CD9 was associated with a significantly increased risk of malignancy, especially when expression was almost completely absent (scores of 0 or $1 ; P<0.0001$ ). Increased expression of CD151, EGFR, and COX2 was similarly associated with malignant transformation. Immunohistochemical scores of greater than 2 and 3 for CD151 and EGFR, respectively, were significant $(P=0.0002$ and 0.006 , respectively). COX2 demonstrated a much more linear effect, as increasing expression correlated with increasing risk of cancer. Her2 and CD82 had no prognostic ability in this cohort 
and indeed demonstrated little expression overall in dysplastic tissues. CD9 and COX2 remained independently prognostic when accounting for the effect of other variables on multivariate analysis ( $P=0.009$ and 0.007 , respectively). When both clinical factors and biomarkers were included in multivariate analysis, the best combination for predicting malignant progression was high dysplasia grade (HR 1.64) strong COX2 staining (HR 1.12), and weak CD9 staining $(P=0.01)$.

As might have been expected, increasing severity of dysplasia and erythroplakic lesions had higher malignant transformation rates on univariate logistic regression $(P=0.0002$ and 0.03 , respectively). The anatomical site within the oral cavity was not prognostic in this cohort, which may in part be explained by the slightly low numbers of known high-risk floor of mouth lesions (13\%), along with the grouping of all tongue lesions together (ventral tongue lesions are known to higher rates of transformation than others). Alcohol and smoking consumption were similarly not prognostic in this cohort. This is in keeping with other studies, where despite these habits increasing the likelihood of developing potentially malignant lesions, they do not appear to increase the subsequent risk of malignant transformation (Napier and Speight, 2008; Liu et al, 2010). Gender was also not independently prognostic; however, females were more at risk when included in a multivariate analysis in combination with histological grade.

Relatively few studies have examined the role of tetraspanins with specific regards to head and neck cancer, and none in OED. However, the findings of these studies support our results. Decreased immunohistochemical expression of CD9 was detected in $42 \%$ of 129 oral cancer samples, with these cases significantly associated with regional nodal metastases $(P=0.017)$ and a reduced overall and disease-free 5-year survival $(P=0.071$ and $P=0.01$, respectively) (Grandis and Tweardy, 1993; Etienne et al, 1999; Ang et al, 2002; Buim et al, 2010). In the same study, $80 \%$ of cases had reduced or absent CD82 staining; however, no correlation with disease-free or overall survival was observed. A study of 34 patients with head and neck cancer identified the same prolongation of overall and disease-free survival $(P=0.02$; $0.004)$ with lower recurrence rates and stage of regional lymphadenopathy $(P=0.02 ; 0.04)$ in cases with increased CD9 expression (Erovic et al, 2003). Loss of CD9 expression has also been noted at the invasive front of tumours (Kusukawa et al, 2001).

CD9 has also been shown to exert an effect on EGFR, with complexes of CD9, EGFR, and $\beta 1$ integrin colocalised in areas of cell-cell interaction. Through EGF induced EGFR receptor internalisation, CD9 attenuates EGFR signalling by reducing cell surface EGFR expression (Murayama et al, 2008). Additional indirect effects on EGFR occur through its receptor ligands. CD9 not only binds to transforming growth factor $\alpha$ (TGF- $\alpha$ ) affecting its cell-surface presentation and distribution, but also prevents its cleavage to produce free ligand. This alteration in EGF receptor stimulation leads ultimately to differences in the effect of receptor activation (Shi et al, 2000; Imhof et al, 2008). These results might suggest that the consequences of decreased CD9 expression in OED are not driven through a direct effect, but through the alteration in balance of EGFR activation. This would be in keeping with the finding from the experiments conducted here, demonstrating increased EGFR expression as a prognostic variable on univariate analysis, despite no obvious direct correlation seen between the expression patterns of the two markers.

Increased CD151 expression is associated with poor prognosis in cancers of the lung (Tokuhara et al, 2001), skin (Woegerbauer et al, 2010), and breast (Yang et al, 2008). Few studies have assessed the role of CD151 in head and neck cancer, and those undertaken show contradictory results. Increased expression of CD151 conferred a significantly poorer prognosis in 73 gingival squamous cell carcinomas (Hirano et al, 2009). However, a recent publication found no prognostic significance of CD151 expression in 83 oral cancer cases, despite the widespread expression of the protein (Romanska et al, 2012). There was a significant association between CD151 and EGFR, both of which were also found at the invasive front along with the $\alpha 3 \beta 1$ integrin (which is also known to form complexes with CD151). The authors suggested that CD151 acts to modulate and coordinate an interaction between EGFR and $\alpha 3 \beta 1$ integrin, even though CD151 was unable to significantly predict malignant progression. In our study, increased expression of CD151 was prognostic on univariate logistic regression, although this effect was lost in a time to event analysis. Furthermore, CD151 did not remain independently prognostic when considered in a multivariate analysis. As with CD9, the effect of CD151 seen in this study and in other cancer types may be driven through its association with EGF receptors and integrins. This is supported by the finding that in CD151 gene-deleted mice expressing erbB2, tumour onset was delayed and cells survival shortened through impaired activation of MAPK-dependent pathways. EGF-mediated cell motility and invasion was also reduced, effects mediated via $\alpha 6 \beta 4$ integrin (Deng et al, 2012). Further study of this tetraspanin is required in OED to identify its true prognostic ability.

Only one study has examined the expression of CD82 in preneoplastic tissue along with the main cohort of OSCC (Uzawa et al, 2002). In 28 leukoplakias (the presence or degree of dysplasia was not reported), decreased CD82 expression was noted in $13(46 \%)$ samples. Decreased expression was also seen in the metastatic OSCC cases in this cohort. This is in keeping with the role of CD82 as a metastasis suppressor. The downregulation in leukoplakias led the authors to postulate that loss of CD82 expression was an early event in the carcinogenic process. In the experiments presented here, $31 \%$ of cases had negative CD82 staining, but no evidence was found that reduced expression is associated with an increased risk of malignant transformation.

Increased COX-2 expression is known to occur in premalignant tissues in many sites, including colon, bladder and stomach (Eberhart et al, 1994; Shirahama, 2000; Sung et al, 2000). Similar upregulation occurs in premalignant lesions and cancers of the head and neck. Cross-sectional studies have all demonstrated an increased expression of COX-2 in premalignant tissue compared with normal mucosa (Shibata et al, 2005). This finding has been replicated in other studies, along with a significant increase in COX-2 expression with increasing severity of dysplasia (Nathan et al, 2001; Banerjee et al, 2002). Despite the interest in COX enzymes as biomarkers in carcinogenesis, until now, no longitudinal studies have examined their role as predictors of malignant transformation of OED in the head and neck. We have demonstrated not only that COX2 has a significant prognostic potential, but also that the risk of malignant transformation appears to escalate with increasing COX2 expression (Figure 4). COX-2 is also a modulator of the EGF pathway. COX-2 expression results in increased levels of $\mathrm{PGE}_{2}$. This prostanoid in turn stimulates EGFR signalling, although the exact mechanisms by which $\mathrm{PGE}_{2}$ activates EGFR have not been fully elucidated (Dannenberg and Subbaramaiah, 2008). Transactivation of the EGF receptor by $\mathrm{PGE}_{2}$ via matrix metalloproteinases and increased release of an EGFR ligand amphiregulin, have also been described (Pai et al, 2002; Buchanan et al, 2003; Shao et al, 2003).

Overexpression of EGFR is known to occur in oral premalignant lesions (Rautava et al, 2008). In contrast to the results presented here, Taoudi Benchekroun et al (2010) examining an cohort of oral premalignant lesions failed to show a statistically significant risk of progression to oral squamous cell cancer in patients with elevated EGFR immunoreactivity, despite high EGFR expression occurring in $71 \%$ of the patients. This disparity may be explained by over two thirds of that particular cohort having a histological diagnosis of hyperplasia only without dysplasia. The prognostic potential of EGFR on univariate analysis in this cohort would support the 
hypothesis of treating these high-risk lesions with EGFR antagonists. Furthermore, evidence is beginning to emerge about the interaction between EGFR and COX2, CD9 and CD151 (Shi et al, 2000; Dannenberg and Subbaramaiah, 2008; Murayama et al, 2008; Deng et al, 2012; Romanska et al, 2012). This also raises the possibility of multimodal approaches to chemoprevention in the management of oral premalignant lesions. Indeed, the finding here that both EGFR and COX-2 are prognostic in OED would support a current phase I/II trial examining the effects of antiEGFR/COX2 in premalignant head and neck lesions (ClinicalTrials.gov identifier NCT00314262). Further work on the role of tetraspanins in OED may help develop targeted therapies against them in the future. New agents are much needed, as neither surgery (the current mainstay of treatment) nor other chemopreventative strategies have so far shown any ability to prevent malignant progression in OED (Lodi et al, 2006). A wider adoption of the REMARK guidelines is important to improve the transparency and reporting of future biomarker studies. This in turn may allow more rapid progress to be made where improving patient selection for selected therapies in OED much needed.

Limitations of the study. Although four of the biomarkers were prognostic in this study, the thresholds identified to differentiate between cases likely or not to progress (CD9, CD151, and EGFR) are data driven, and therefore possibly unique to this data set. In this respect, the results must be viewed with caution and are perhaps best considered as representing a hypothesis-generating group. A validation cohort would be required to test these thresholds. Furthermore, it was not possible to construct a prognostic classifier based on the numbers in this study and so any validation cohort would need to be larger to enable this.

Despite being one of the largest cohorts of oral dysplasia used to date in assessing the prognostic ability of biomarkers, there remain the same limitations such as inadequate data collection and variability in the treatment of similar lesions from individuals at different institutions that affect all retrospectively collected cohorts. As an example of this, while some studies have reported higher transformation rates of oral leukoplakia in females and from particular anatomical areas (lateral border of tongue and floor of mouth) other recent large cohort studies have similarly to here failed to demonstrate this (Holmstrup et al, 2006; Napier and Speight, 2008; Liu et al, 2011). It is possible that this difference may be explained in part because of difference in cohorts (e.g., in this study all cases were OED, whereas in others leukoplakia without dysplasia was also included). Poor clinical recording did not allow a sub-site analysis of lesions of the tongue to be performed, meaning all cases affecting the tongue (the largest site numerically) were analysed together, potentially obscuring a significant effect of anatomical site. These limitations may only be improved by the prospective enrollment of patients with OED into clinical trials.

Conclusions. This study, using one of the largest multicentre cohorts of OED in the literature, demonstrates four biomarkers (EGFR, CD151, CD9, and COX2) with a prognostic ability. It is also the first study to examine both the expression and the prognostic ability of the tetraspanins in OED. If validated, then these results may help improve identification of those patients at highest risk of malignant transformation and also suggests other avenues for chemoprevention and chemotherapeutics in the treatment of this condition.

\section{ACKNOWLEDGEMENTS}

PN was supported by research fellowships from the Royal College of Surgeons of England and University Hospital Coventry and Warwickshire. PHR received funding from Yorkshire Cancer Research grant number L341PG. We gratefully acknowledge the contribution to this study made by the University of Birmingham's Human Biomaterials Resource Centre.

\section{CONFLICT OF INTEREST}

The authors declare no conflicts of interest.

\section{REFERENCES}

Abbey LM, Kaugars GE, Gunsolley JC, Burns JC, Page DG, Svirsky JA, Eisenberg E, Krutchkoff DJ, Cushing M (1995) Intraexaminer and interexaminer reliability in the diagnosis of oral epithelial dysplasia. Oral Surg Oral Med Oral Pathol Oral Radiol Endod 80: 188-191.

Ang KK, Berkey BA, Tu X, Zhang H-Z, Katz R, Hammond EH, Fu KK, Milas L (2002) Impact of epidermal growth factor receptor expression on survival and pattern of relapse in patients with advanced head and neck carcinoma. Cancer Res 62: 7350-7356.

Banerjee AG, Gopalakrishnan VK, Bhattacharya I, Vishwanatha JK (2002) Deregulated cyclooxygenase-2 expression in oral premalignant tissues. Mol Cancer Ther 1: 1265-1271.

Barnes L, Eveson JW, Reichart P, Sidransky D (eds) (2005) World Health Organization Classification of Tumours, Pathology and Genetics of Head and Neck Tumours. IARC Press: Lyon.

Berditchevski F (2001) Complexes of tetraspanins with integrins: more than meets the eye. J Cell Sci 114: 4143-4151.

Buchanan F, Wang D, Bargiacchi F, DuBois R (2003) Prostaglandin E2 regulates cell migration via the intracellular activation of the epidermal growth factor receptor. J Biol Chem 278: 35451-35457.

Buim MEC, Lourenço SV, Carvalho KC, Cardim R, Pereira C, Carvalho AL, Fregnani JH, Soares FA (2010) Downregulation of CD9 protein expression is associated with aggressive behavior of oral squamous cell carcinoma. Oral Oncol 46: 166-171.

Califano J, der Riet, van P, Westra W, Nawroz H, Clayman G, Piantadosi S, Corio R, Lee D, Greenberg B, Koch W, Sidransky D (1996) Genetic progression model for head and neck cancer: implications for field cancerization. Cancer Res 56: 2488-2492.

Carrasco JL, Jover L (2003) Estimating the generalized concordance correlation coefficient through variance components. Biometrics 59: $849-858$.

Dannenberg A, Subbaramaiah K (2008) Crosstalk between COX-2 and EGFR: a potential therapeutic opportunity. EGFR Signaling Networks in Cancer Therapy 325-339.

Deng X, Li Q, Hoff J, Novak M, Yang H, Jin H, Erfani SF, Sharma C, Zhou P, Rabinovitz I, Sonnenberg A, Yi Y, Zhou P, Stipp CS, Kaetzel DM, Hemler ME, Yang XH (2012) Integrin-associated CD151 drives ErbB2evoked mammary tumor onset and metastasis. Neoplasia 14: 678-689.

Eberhart CE, Coffey RJ, Radhika A, Giardiello FM, Ferrenbach S, DuBois RN (1994) Up-regulation of cyclooxygenase 2 gene expression in human colorectal adenomas and adenocarcinomas. Gastroenterology 107: $1183-1188$.

Erovic BM, Pammer J, Hollemann D, Woegerbauer M, Geleff S, Fischer MB, Burian M, Frommlet F, Neuchrist C (2003) Motility-related protein-1/ CD9 expression in head and neck squamous cell carcinoma. Head Neck 25: 848-857.

Etienne MC, Pivot X, Formento JL, Bensadoun RJ, Formento P, Dassonville O, Francoual M, Poissonnet G, Fontana X, Schneider M, Demard F, Milano G (1999) A multifactorial approach including tumoural epidermal growth factor receptor, p53, thymidylate synthase and dihydropyrimidine dehydrogenase to predict treatment outcome in head and neck cancer patients receiving 5-fluorouracil. Br J Cancer 79: 1864-1869.

Farhadieh RD, Smee R, Ow K, Yang JL, Russell PJ, Crouch R, Jackson P, Jacobson IV (2004) Down-regulation of KAI1/CD82 protein expression in oral cancer correlates with reduced disease free survival and overall patient survival. Cancer Lett 213: 91-98.

Ferlay J, Shin H-R, Bray F, Forman D, Mathers C, Parkin DM (2010) Estimates of worldwide burden of cancer in 2008: GLOBOCAN 2008. Int J Cancer 127: 2893-2917.

Fischer DJ, Epstein JB, Morton TH, Schwartz SM (2004) Interobserver reliability in the histopathologic diagnosis of oral pre-malignant and malignant lesions. J Oral Pathol Med 33: 65-70. 
Goldstein NS, Bosler D (2007) An approach to interpreting immunohistochemical stains of adenocarcinoma in small needle core biopsy specimens: the impact of limited specimen size. Am J Clin Path 127: 273-281.

Grandis JR, Tweardy DJ (1993) Elevated levels of transforming growth factor alpha and epidermal growth factor receptor messenger RNA are early markers of carcinogenesis in head and neck cancer. Cancer Res 53: 3579-3584.

Hemler ME (2005) Tetraspanin functions and associated microdomains. Nat Rev Mol Cell Biol 6: 801-811.

Hirano C, Nagata M, Noman AA, Kitamura N, Ohnishi M, Ohyama T, Kobayashi T, Suzuki K, Yoshizawa M, Izumi N, Fujita H, Takagi R (2009) Tetraspanin gene expression levels as potential biomarkers for malignancy of gingival squamous cell carcinoma. Int J Cancer 124: 2911-2916.

Hogmo A, Lindskog S, Lindholm J, Kuylenstierna R, Auer G, Munck-Wikland E (1998) Preneoplastic oral lesions: the clinical value of image cytometry DNA analysis, p53 and p21/WAF1 expression. Anticancer Res 18: 3645-3650.

Holmstrup P, Vedtofte P, Reibel J, Stoltze K (2006) Long-term treatment outcome of oral premalignant lesions. Oral Oncol 42: 461-474.

Hsue SS, Wang WC, Chen CH, Lin CC, Chen YK, Lin LM (2007) Malignant transformation in 1458 patients with potentially malignant oral mucosal disorders: a follow-up study based in a Taiwanese hospital. J Oral Pathol Med 36: 25-29.

Imai Y, Sasaki T, Shinagawa Y, Akimoto K, Fujibayashi T (2002) Expression of metastasis suppressor gene (KAI1/CD82) in oral squamous cell carcinoma and its clinico-pathological significance. Oral Oncol 38: $557-561$.

Imhof I, Gasper WJ, Derynck R (2008) Association of tetraspanin CD9 with transmembrane TGF alpha\} confers alterations in cell-surface presentation of TGF $\{$ alpha\} and cytoskeletal organization. J Cell Sci 121: 2265-2274.

Karabulut A, Reibel J, Therkildsen MH, Prætorius F, Nielsen HW, Dabelsteen E (1995) Observer variability in the histologic assessment of oral premalignant lesions. J Oral Pathol Med 24: 198-200.

Kirkegaard T, Edwards J, Tovey S, McGlynn LM, Krishna SN, Mukherjee R, Tam L, Munro AF, Dunne B, Bartlett JMS (2006) Observer variation in immunohistochemical analysis of protein expression, time for a change? Histopathology 48: 787-794.

Kusukawa J, Ryu F, Kameyama T, Mekada E (2001) Reduced expression of CD9 in oral squamous cell carcinoma: CD9 expression inversely related to high prevalence of lymph node metastasis. J Oral Pathol Med 30: 73-79.

Landis JR, Koch GG (1977) The measurement of observer agreement for categorical data. Biometrics 33: 159-174.

Lazo PA (2007) Functional implications of tetraspanin proteins in cancer biology. Cancer Sci 98: 1666-1677.

Little KD, Hemler ME, Stipp CS (2004) Dynamic regulation of a GPCRtetraspanin-G protein complex on intact cells: central role of CD81 in facilitating GPR56-Galpha q/11 association. Mol Biol Cell 15: 2375-2387.

Liu W, Bao Z-X, Shi L-J, Tang G-Y, Zhou Z-T (2011) Malignant transformation of oral epithelial dysplasia: clinicopathological risk factors and outcome analysis in a retrospective cohort of 138 cases. Histopathology 59: 733-740.

Liu W, Wang Y-F, Zhou H-W, Shi P, Zhou Z-T, Tang G-Y (2010) Malignant transformation of oral leukoplakia: a retrospective cohort study of 218 Chinese patients. BMC Cancer 10: 685.

Lodi G, Sardella A, Bez C, Demarosi F, Carrassi A (2006) Interventions for treating oral leukoplakia. Cochrane Database Syst Rev (6): doi:10.1002/ 14651858.CD001829.pub3.

McShane LM, Altman DG, Sauerbrei W, Taube SE, Gion M, Clark GM. Statistics Subcommittee of the NCI-EORTC Working Group on Cancer Diagnostics (2005) REporting recommendations for tumour MARKer prognostic studies (REMARK). Br J Cancer 93: 387-391.

Mehanna HM, Rattay T, Smith J, McConkey CC (2009) Treatment and follow-up of oral dysplasia - a systematic review and meta-analysis. Head Neck 31: 1600-1609.

Mhawech P, Dulguerov P, Tschanz E, Verdan C, Ares C, Allal AS (2004) Motility-related protein-1 (MRP-1/CD9) expression can predict diseasefree survival in patients with squamous cell carcinoma of the head and neck. Br J Cancer 90: 471-475.

Murayama Y, Shinomura Y, Oritani K, Miyagawa J-I, Yoshida H, Nishida M, Katsube F, Shiraga M, Miyazaki T, Nakamoto T, Tsutsui S, Tamura S, Higashiyama S, Shimomura I, Hayashi N (2008) The tetraspanin CD9 modulates epidermal growth factor receptor signaling in cancer cells. J Cell Physiol 216: 135-143.

Muzio Lo,L, Pannone G, Leonardi R, Staibano S, Mignogna MD, De Rosa G, Kudo Y, Takata T, Altieri DC (2003) Survivin, a potential early predictor of tumor progression in the oral mucosa. J Dental Res 82: 923-928.

Nankivell P, Mehanna H (2011) Oral dysplasia: biomarkers, treatment, and follow-up. Curr Oncol Rep 13: 145-152.

Nankivell PC, Williams H, Bartlett JMS, Mehanna H (2012) Validation of tissue microarrays in oral epithelial dysplasia using a novel virtual-array technique. J Clin Pathol 65: 1084-1087.

Napier SS, Speight PM (2008) Natural history of potentially malignant oral lesions and conditions: an overview of the literature. J Oral Pathol Med 37: $1-10$.

Nathan CA, Leskov IL, Lin M, Abreo FW, Shi R, Hartman GH, Glass J (2001) COX-2 expression in dysplasia of the head and neck: correlation with elF4E. Cancer 92: 1888-1895.

Normanno N, Bianco C, Luca A, Strizzi L, Gallo M, Mancino M, Salomon D (2008) Expression and prognostic significance of the EGFR in solid tumors. EGFR Signal Networks Cancer Ther 210-223.

Odintsova E, van Niel G, Conjeaud H, Raposo G, Iwamoto R, Mekada E, Berditchevski F (2013) Metastasis suppressor tetraspanin CD82/KAI1 regulates ubiquitylation of epidermal growth factor receptor. J Biol Chem 288(36): 26323-26334.

Pai R, Soreghan B, Szabo IL, Pavelka M, Baatar D, Tarnawski AS (2002) Prostaglandin E2 transactivates EGF receptor: a novel mechanism for promoting colon cancer growth and gastrointestinal hypertrophy. Nat Med 8: 289-293.

Park S-Y, Yoon S-J, Freire-de-Lima L, Kim J-H, Hakomori S-I (2009) Control of cell motility by interaction of gangliosides, tetraspanins, and epidermal growth factor receptor in A431 versus KB epidermoid tumor cells. Carbohydr Res 344: 1479-1486.

Rautava J, Jee KJ, Miettinen PJ, Nagy B, Myllykangas S, Odell EW, Soukka T, Morgan PR, Heikinheimo K (2008) ERBB receptors in developing, dysplastic and malignant oral epithelia. Oral Oncol 44: $227-235$.

Romanska HM, Potemski P, Collins SI, Williams H, Parmar S, Berditchevski F (2012) Loss of CD151/Tspan24 from the complex with integrin $\hat{\mathrm{I}} \pm 3 \hat{\mathrm{I}}^{2} 1$ in invasive front of the tumour is a negative predictor of disease-free survival in oral squamous cell carcinoma. Oral Oncol 1-6.

Rosin MP, Cheng X, Poh C, Lam WL, Huang Y, Lovas J, Berean K, Epstein JB, Priddy R, Le ND, Zhang L (2000) Use of allelic loss to predict malignant risk for low-grade oral epithelial dysplasia. Clin Cancer Res 6 : 357-362.

Shao J, Lee S, Guo H, Evers B, Sheng H (2003) Prostaglandin E2 stimulates the growth of colon cancer cells via induction of amphiregulin. Cancer Res 63: 5218-5223.

Shi W, Fan H, Shum L, Derynck R (2000) The tetraspanin CD9 associates with transmembrane TGF-alpha and regulates TGF-alpha-induced EGF receptor activation and cell proliferation. J Cell Biol 148: 591-602.

Shibata M, Kodani I, Osaki M, Araki K, Adachi H, Ryoke K, Ito H (2005) Cyclo-oxygenase-1 and -2 expression in human oral mucosa, dysplasias and squamous cell carcinomas and their pathological significance. Oral Oncol 41: 304-312.

Shirahama T (2000) Cyclooxygenase-2 expression is up-regulated in transitional cell carcinoma and its preneoplastic lesions in the human urinary bladder. Clin Cancer Res 6: 2424-2430.

Silverman S, Gorsky M, Lozada F (1984) Oral leukoplakia and malignant transformation. A follow-up study of 257 patients. Cancer 53: 563-568.

Smith J, Rattay T, Mcconkey C, Helliwell T, Mehanna H (2009) Biomarkers in dysplasia of the oral cavity: a systematic review. Oral Oncol 45: 647-653.

Sung JJ, Leung WK, Go MY, To KF, Cheng AS, Ng EK, Chan FK (2000) Cyclooxygenase-2 expression in Helicobacter pylori-associated premalignant and malignant gastric lesions. Am J Pathol 157: 729-735.

Tabor MP, Braakhuis BJM, van der Wal JE, van Diest PJ, Leemans CR, Brakenhoff RH, Kummer JA (2003) Comparative molecular and histological grading of epithelial dysplasia of the oral cavity and the oropharynx. J Pathol 199: 354-360.

Taoudi Benchekroun M, Saintigny P, Thomas SM, El-Naggar AK, Papadimitrakopoulou V, Ren H, Lang W, Fan Y-H, Huang J, Feng L, Lee JJ, Kim ES, Hong WK, Johnson FM, Grandis JR, Mao L (2010) Epidermal growth factor receptor expression and gene copy number in the risk of oral cancer. Cancer Prev Res 3: 800-809. 
Tokuhara T, Hasegawa H, Hattori N, Ishida H, Taki T, Tachibana S, Sasaki S, Miyake M (2001) Clinical significance of CD151 gene expression in non-small cell lung cancer. Clin Cancer Res 7: 4109-4114.

Uzawa K, Ono K, Suzuki H, Tanaka C, Yakushiji T, Yamamoto N, Yokoe H, Tanzawa H (2002) High prevalence of decreased expression of KAI1 metastasis suppressor in human oral carcinogenesis. Clin Cancer Res 8: $828-835$.

Wang X-Q, Yan Q, Sun P, Liu J-W, Go L, McDaniel SM, Paller AS (2007) Suppression of epidermal growth factor receptor signaling by protein kinase C-alpha activation requires CD82, caveolin-1, and ganglioside. Cancer Res 67: 9986-9995.

Warnakulasuriya S, Reibel J, Bouquot J, Dabelsteen E (2008) Oral epithelial dysplasia classification systems: predictive value, utility, weaknesses and scope for improvement. J Oral Pathol Med 37(3): 127-133.

Woegerbauer M, Thurnher D, Houben R, Pammer J, Kloimstein P, Heiduschka G, Petzelbauer P, Erovic BM (2010) Expression of the tetraspanins CD9, CD37, CD63, and CD151 in Merkel cell carcinoma: strong evidence for a posttranscriptional fine-tuning of CD9 gene expression. Mod Pathol 23: 751-762.
Yang XH, Richardson AL, Torres-Arzayus MI, Zhou P, Sharma C, Kazarov AR, Andzelm MM, Strominger JL, Brown M, Hemler ME (2008) CD151 accelerates breast cancer by regulating alpha 6 integrin function, signaling, and molecular organization. Cancer Res 68: 3204-3213.

Zhang L, Poh CF, Williams M, Laronde DM, Berean K, Gardner PJ, Jiang H, Wu L, Lee JJ, Rosin MP (2012) Loss of heterozygosity (loh) profiles-validated risk predictors for progression to oral cancer. Cancer Prev Res 5: 1081-1089.

Zhou X, Jordan RCK, Li Y, Huang B-L, Wong DTW (2005) Frequent allelic imbalances at $8 \mathrm{p}$ and 11q22 in oral and oropharyngeal epithelial dysplastic lesions. Cancer Genet Cytogenet 161: 86-89.

Zöller M (2009) Tetraspanins: push and pull in suppressing and promoting metastasis. Nat Rev Cancer 9: 40-55.

This work is published under the standard license to publish agreement. After 12 months the work will become freely available and the license terms will switch to a Creative Commons AttributionNonCommercial-Share Alike 3.0 Unported License.

Supplementary Information accompanies this paper on British Journal of Cancer website (http://www.nature.com/bjc) 